\title{
Publisher Correction: Bitcoin emissions alone could push global warming above $2{ }^{\circ} \mathrm{C}$
}

Camilo Mora, Randi L. Rollins, Katie Taladay, Michael B. Kantar, Mason K. Chock, Mio Shimada and Erik C. Franklin

Correction to: Nature Climate Change https://doi.org/10.1038/s41558-018-0321-8, published online 29 October 2018.

In the version of this Comment originally published, the last year on the axis of Fig. 1c read 3000; it should have read 2100 . This has now been corrected. 